
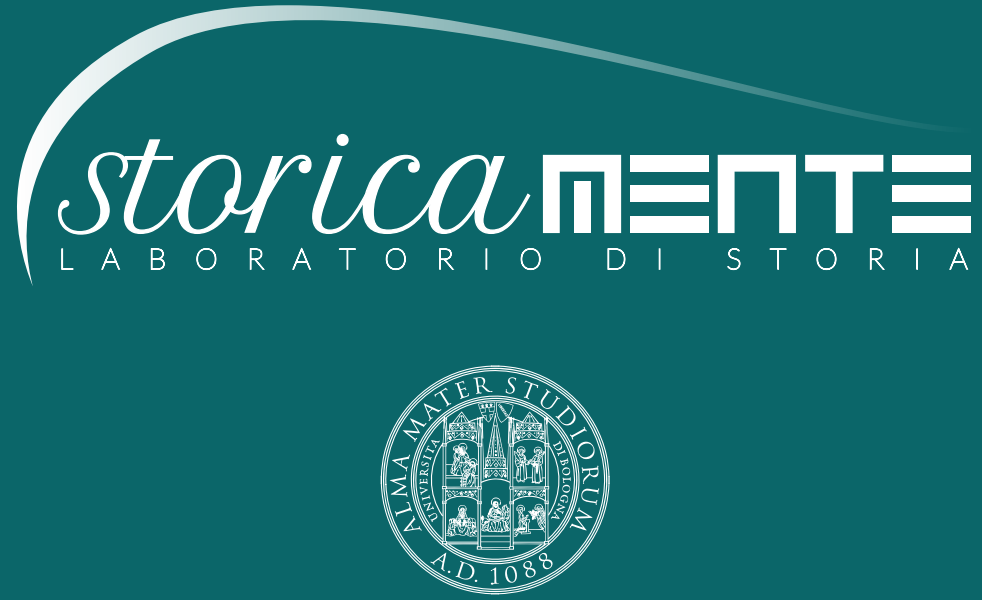

ALMA MATER STUDIORUM

Università di Bologna

Dipartimento di Storia Culture Civiltà

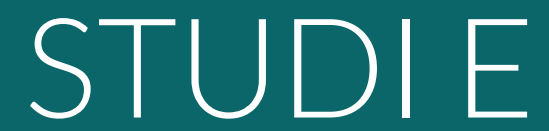

RICERCHE 


\section{STORICAMENTE.ORG}

\section{Laboratorio di Storia}

\section{Sara Valentina Di Palma}

Corpi di donne in guerra. La violenza sessuale in Bosnia e Ruanda e i problemi del dopoguerra

Numero 10 - 2014

ISSN: $1825-411 \mathrm{X}$

Art. 2

pp. 1-35

DOI: $10.12977 /$ stor508

Editore: BraDypUS

Data di pubblicazione: 14/06/2014

Sezione: Studi e Ricerche

Questo articolo è stato sottoposto a un processo di double blind peer review 


\title{
Corpi di donne in guerra. La violenza sessuale in Bosnia e Ruanda e i problemi del dopoguerra
}

\author{
SARA VALENTINA DI PALMA \\ Univ. Siena, Dipartimento Scienze sociali, \\ politiche e cognitive
}

L'articolo analizza la violenza sessuale nei conflitti in Bosnia e Ruanda durante gli anni '90. In primo luogo, si discuterà le locuzione di "conflitti etnici" e si mostrerà come la definizione etnica di queste guerre sia fuorviante, in quanto essa occulta la costruzione di un nazionalismo basato sull'uso del corpo femminile come simbolo della nazione. In secondo luogo si esaminerà come, sia in Bosnia sia in Ruanda, la violenza si incentrò non solo sui corpi delle donne, ma collegò nazionalismo, genere, corpo sessuato e sessualità allo scopo di compromettere il futuro del gruppo nemico nel dopoguerra. In terzo luogo, mi limiterò a descrivere come nei postumi dei conflitti i programmi di recupero non sono adeguati, escludendo molti sopravvissuti, che sono emarginati, stigmatizzati, messi sotto silenzio, e quindi sottoposti a nuove violenze.

This article studies sexual violence in the 1990s conflicts in Bosnia and Rwanda. First I will show how the ethnic definition is misleading, since it hides what is really the construction of nationalism, implemented through the use of the female body as a symbol of the nation. Second, I will examine how this violence interconnects nationalism, gender, gendered-body and sexuality with the aim of affecting the future of the enemy group. Thirdly, I will describe how, in the conflicts' aftermaths, recovery programmes are not adequate and exclude many survivors, who are marginalized, stigmatized and silenced and thus subjected to new violence. 


\section{Introduzione}

Questo articolo si occupa della violenza sessuale, incluso lo stupro di massa, nei conflitti degli anni Novanta in Bosnia' e in Ruanda, e della situazione postbellica. I due case studies ${ }^{2}$ sono interessanti per la strumentalizzazione del corpo femminile allo scopo di annientare il nemico, in una competizione politica di movimenti nazionalisti i quali prendono piede attraverso la sessualizzazione dei cittadini: uomini combattenti per la patria versus donne silenziose, complici o nemiche da annientare [Richter 2003, 35].

Queste guerre sono state spesso definite “conflitti etnici”, una definizione che sarà qui discussa contestualizzando la preparazione di entrambe in relazione alla violenza sessuale e al suo rapporto con il nazionalismo; analizzando quindi quanto accadde durante le guerre stesse; e infine descrivendo le conseguenze della violenza nel dopoguerra di entrambi i paesi.

La comparazione tra i due case studies nasce da diverse considerazioni: entrambi sono stati genericamente inclusi tra le "nuove guerre" degli anni Novanta (un'altra etichetta che sarà analizzata in questa sede) in cui la grande copertura mediatica dei conflitti ha denunciato gli stupri di massa e il loro uso come arma bellica, senza comprenderne però appieno la portata in termini di intenzionalità a colpire non solo il gruppo

"Bosnia": abbreviazione comunemente usata per lo Stato di "Bosnia e Herzegovina”. Gli accordi di Dayton del 1995, a seguito della guerra di dissoluzione della Jugoslavia, hanno previsto la divisione di questo Stato, ex Repubblica Federale Socialista Jugoslava di Bosnia e Herzegovina, in due entità dall'ampia autonomia ma ancora prive di personalità giuridica internazionale e vincolate dal veto parlamentare dell'Alto Commissariato ONU, la Federazione Bosnia e Herzegovina (abitata soprattutto da musulmani di Bosnia e croati) e la Republika Srpska (abitata prevalentemente da serbi), oltre al piccolo distretto di Brčko (entità amministrativa autonoma con controllo internazionale, unica eccezione a Dayton).

2 Sulle guerre balcaniche Woodward 1995, Rieff 1996 e Marzo Magno 2001; sul genocidio rwandese Sibomana 1998, Gourevitch 2000, Dallaire 2003, Grill 2005 e Scaglione 2010 . 
nemico ma anche il suo possibile recupero postbellico e il nuovo uso del corpo delle donne in questa strategia bellica. In entrambi i casi, inoltre, sono stati istituiti due tribunali internazionali ad hoc che hanno compiuto importanti passi avanti a livello giurisprudenziale nella definizione di stupro di guerra e nel tentativo di tutelarne i bersagli [Gaeta 2006], anche se ambedue i tribunali hanno incontrato poi grandi problemi in sede processuale e nel progressivo affidamento dei casi minori alle corti locali. Entrambi i case studies mostrano poi la discrepanza tra una giurisprudenza innovativa a livello internazionale e l'incapacità delle Nazioni Unite e degli altri attori internazionali prima a prevenire e poi ad affrontare adeguatamente le crisi in corso [Sekulić 2006]; infine sia in Bosnia sia in Ruanda il dopoguerra ha comportato una difficile ricostruzione a causa del parziale riconoscimento delle vittime o del loro sfruttamento nel nuovo modello nazionalista.

In primo luogo, si discuteranno le locuzioni di "nuove guerre" e di "conflitti etnici" e si mostrerà come la definizione etnica di queste guerre sia fuorviante, in quanto essa occulta la costruzione di un nazionalismo basato sull'uso del corpo femminile come simbolo della nazione. Secondariamente si esaminerà come, sia in Bosnia sia in Ruanda, la violenza - che fu non solo sessuale e fisica, ma anche psicologica e simbolica - si incentrò non solo sui corpi delle donne, ma collegò nazionalismo, genere, corpo sessuato e sessualità allo scopo di compromettere il futuro del gruppo nemico nel dopoguerra. La ricerca si occupa di questi due case studies per mostrare come il nazionalismo fu sessualizzato in entrambi i paesi, e come "collegare l'etnicità al genere e all'eterosessualità rende possibile una nuova concettualizzazione del nazionalismo» [Žarkov 2007, 11]. Il saggio descriverà infine come, nel dopoguerra di entrambi i conflitti, i programmi di ricostruzione postbellica furono inadeguati ed esclusero molti sopravvissuti alla violenza sessuale, i quali furono marginalizzati, stigmatizzati e messi a tacere esponendoli così a una nuova violenza psicologica e fisica - nel momento, come vedremo, in cui spesso fu limitato loro l'accesso alle cure mediche e psicologiche, mentre 
vecchi e nuovi perpetratori li sottoposero a ulteriori vessazioni. Questo lavoro fa propria la messa in discussione dell'attenzione di una parte del post-femminismo alla sola interiorità femminile e alla psicologia delle vittime di stupro cristallizzata in un paradigma interpretativo statico, e sposa invece la tesi femminista che vede la violenza sessuale come un fenomeno in cui la donna non è soltanto una vittima passiva. Secondo un certo post-femminismo, infatti, la prospettiva e la narrativa femminile appaiono immutabili nel tempo e lo stupro è visto solo come legato alla violazione dell'integrità della donna [Marcus 1992; Brown 1995], senza considerarlo anche una violazione dell'onore della famiglia e della comunità. Si ritiene qui inoltre che la parola "vittima" debba essere riconsiderata in relazione all'esperienza della donna non solo durante lo stupro ma anche dopo, tenendo presenti gli aspetti politici, sociali e psicologici della violenza, della sopravvivenza e del recupero [Mardorossian 2002, 747-48, 754]. Le donne possono infatti trovare proprie strategie di reazione, e considerare l'esperienza della violenza sessuale che hanno vissuto non solo secondo le categorie di "vittima" e di "sopravvissuta". Tale discorso si lega direttamente alla questione dello sfruttamento delle storie di violenza sessuale all'interno della nuova memoria storica postbellica, affrontata in conclusione del testo.

Questo saggio guarda infine alle interconnessioni tra genere e sessualità, etnicità e nazionalismo, identità e memoria nel dopoguerra, analizzando le memorie di donne sopravvissute allo stupro di massa e ad altre violenze sessuali e poi di nuovo vittimizzate dalle società postbelliche. Queste donne, intervistate da altre donne e da associazioni femminili e femministe consapevoli dell'uso nazionalistico delle donne prima, durante e dopo la guerra, raccontano una storia di violenza ma anche di reazione. Diversi gruppi hanno iniziato infatti a riflettere sui ruoli della donna nel dopoguerra secondo una nuova «filosofia del femminismo» [Kesić 2003, 49], in cui il racconto della violenza subita riveste un forte significato politico contro l'imposizione al silenzio o contro la manipolazione della memoria femminile al fine di revittimizzare le donne o per sfruttarne la 
vicenda nel nuovo nazionalismo del dopoguerra.

Questo articolo, tenuto conto del dibattito internazionale sulla definizione di stupro in guerra e sui diritti umani delle donne [Vitucci 2007; Flores 2010], usa una definizione ampia di violenza sessuale, includendo in essa ogni tipo di attacco fisico e psicologico, secondo quanto stabilito dalla giurisprudenza internazionale con il processo Akayesu presso il Tribunale Penale Internazionale di Arusha (International Criminal Tribunal for Ruanda, ICTR) nel 1998 allo scopo di evitare l'ambiguità delle diverse interpretazioni legali e culturali, mutevoli nel tempo e nelle società che le utilizzano [Bourke 2007, 405-409; Green 2004, 102] . Viene qui dunque sposata la tesi secondo cui

il Tribunale considera lo stupro come forma di aggressione, i cui elementi centrali non possono essere ridotti alla descrizione meccanica di oggetti usati e di parti del corpo. [...] La violenza sessuale non si limita all'attacco fisico al corpo umano, e può includere atti che non implicano la penetrazione o persino il contatto fisico. [...] Minacce, intimidazioni, estorsioni e altre forme di attacco che provochino paura o disperazione possono costituire coercizione, e la coercizione può verificarsi in alcune circostanze, come i conflitti armati [ICTR-96-4-T, parr. 687 e 688, 167].

\section{La violenza sessuale nelle "nuove guerre" $\mathrm{e}$ nei "conflitti etnici"}

Nel corso del XX secolo, la popolazione civile è stata sempre più coinvolta nei conflitti e attaccata intenzionalmente, secondo una strategia volta a colpire le società nemiche. Attaccare le donne, in particolare,

Tale linea interpretativa è stata poi adottata dallo Statuto della Corte Penale Internazionale a Roma il 17 luglio 1998 (Rome Statute of the ICC, 4). Solo nel 2008 il Consiglio di Sicurezza delle Nazioni Unite ha riconosciuto la definizione legale di stupro in guerra, inteso come "a tactic of war to humiliate, dominate, instil fear in, disperse and/or forcibly relocate civilian members of a community or ethnic group». [United Nations Securi 
è divenuto sempre più comune allo scopo di umiliare il nemico attraverso il corpo delle donne, intaccando così l'autostima del nemico e la sua confidenza nella capacità di proteggere le proprie donne [Diken e Laustsen 2005]. Il Novecento ha vissuto paradossalmente, accanto a una crescente sensibilità per i diritti delle donne [Bartoloni 2002; Rossi-Doria 2004; Salvatici 2007] di cui la IV Conferenza Mondiale delle Donne di Pechino nel 1995 è solo un esempio, la loro negazione de facto: le donne continuano ad essere anzi sempre più spesso «trofei e target di guerra» [Agosín 2001, 2] e la divisione tra sfera pubblica e privata mina l'efficacia degli interventi in loro difesa [Romani 1994)].

Diverse forme di violenza contro le donne, fino allo stupro, sono state usate come mezzo di genocidio contro minoranze, di snazionalizzazione e in politiche di estirpazione di popoli e di deportazione, come mostrano diverse ricerche recenti sul genocidio armeno condotto nel corso del Primo conflitto mondiale dall'esercito turco [Sanasarian 1989], in cui l'umiliazione sessuale fu usata prima per intimidire e poi per assimilare donne armene come mezzo di genocidio [Derderian 2005, 8]. Di nuovo, durante la Prima Guerra Mondiale l'esercito bulgaro stuprò donne serbe nel processo di snazionalizzazione del popolo serbo [Bianchi 2008], e anche in seguito stuprare la donna del nemico fu usata come tattica e non solo per vendetta come in Germania nel 1945 [Beevor 2007, 419-435], o per disonorare il nemico come durante la Partizione tra India e Pakistan [Menon e Bhasin 1998, 41; Menon 2004]. Lo stupro di massa divenne persino un'arma di guerra con l'occupazione dell'armata giapponese a Nanchino nel 1937 [Chang 1998, 85-98].

Dalla fine del Secondo conflitto mondiale, soprattutto con l'aumento dei cosiddetti conflitti asimmetrici (in cui eserciti regolari o truppe paramilitari attaccano i civili e non altri soldati), lo stupro di massa in guerra è aumentato, facendo delle donne le vittime intenzionali delle guerre. Tale processo è culminato con le cosiddette "nuove guerre" [Kaldor 1999], definizione applicata ai conflitti successivi alla Guerra fredda, quando l'instabilità mondiale degli anni Novanta fu caratterizzata da diverse 
guerre locali e regionali, in cui un nazionalismo in crescita e le cosiddette "guerre etniche" comportarono una ridefinizione dell'identità di gruppi di persone in competizione per il potere e per le risorse, un incremento delle vittime civili a causa di un mutato rapporto tra civili e militari, e un nuovo ruolo degli eserciti professionali. I concetti sia di "guerre etniche" sia di "nuove guerre" sono tuttavia problematici. La teoria delle nuove guerre è stata messa in discussione su base quantitativa contestando il fatto che il numero di vittime civili sia cresciuto dopo il 1989 [Melander, Öberg e Hall 2009], e mentre potrebbe essere valida per alcune aree come i Balcani, essa non rende tuttavia conto di altri tipi di conflitto in altre zone del mondo [Newman 2004]; essa inoltre è insensibile alla questione di genere, in quanto non tiene in considerazione la crescente violenza contro le donne in guerra [Copelon 1995] oggetto di questo saggio.

\section{La sessualizzazione dei corpi femminili in Bosnia e in Ruanda}

Guardando a concetti come il primitivismo e il tribalismo [Vidal 1996, 326], si è tentati dall'usare la definizione etnica anche per i conflitti in oggetto in questo studio. La guerra di dissoluzione della ex Jugoslavia (1991-1995) ricevette da subito una grande attenzione per i suoi "stupri etnici”, e ugualmente diverse analisi del genocidio rwandese hanno sottolineato la questione etnica [Bruneteau 2004, 218-220] e la «trappola tribale» [Glover 2001, 123].

Si tratta in realtà di una distorsione linguistica [Macedo 2000, 21]: l'etichetta etnica, infatti, non spiega entrambi i casi, caratterizzati il primo da un aggressivo nazionalismo per dividere la Bosnia [Sekulić 2002; Eadem 2006], e da una lotta per il potere politico ed economico attraverso politiche nazionaliste il secondo. Il terreno per il genocidio rwandese del 1994 fu preparato in precedenza dalla guerra civile (1990-1994) con una intimidazione intra-etnica [Strauss 2006], e con la creazione artificiale 
di confini e la competizione su campi, colture e bestiame [Tilly 2003, 31-32, 136-142], mentre la dissoluzione della ex Jugoslavia dipese dal crescente nazionalismo negli anni Ottanta a causa soprattutto della crisi dell'economia e poi del collasso economico causato da contraddizioni emerse con il crollo del comunismo, dall'inefficienza della macchina statale, dalla crescente competizione tra le Repubbliche della Federazione, dall'accrescersi delle contrapposizioni socioeconomiche tra mondo contadino e proletario, rurale e montano da un lato e realtà borghesi urbane dall'altro [Ramet 2006, 285-323, Rumiz 2011, 102].

In entrambi i paesi, l'uso massiccio della violenza sessuale in guerra fu preparato dalla costruzione prebellica del nazionalismo attraverso politiche di creazione d'identità e di definizione del nemico. La costruzione politica del nazionalismo serbo negli anni Ottanta, in particolare, definì la Serbia in opposizione a un "altro" esterno da cui differenziarsi attraverso simboli e metafore aventi come oggetto il corpo della nazione versus il corpo esterno dei nemici. La violazione di un popolo passa attraverso la violazione delle proprie donne, e questo spiega perché, dopo la morte del presidente della Repubblica Federale Socialista di Jugoslavia, Josip Broz Tito, nel 1980, crebbero le accuse (false) di stupri perpetrati dagli albanesi del Kosovo (una delle due provincie autonome della Repubblica di Serbia, dichiaratasi unilateralmente indipendente dalla Serbia nel 2008 senza che la Serbia a oggi la riconosca) contro donne, e persino contro uomini - lo stupro come possesso e umiliazione del corpo del nemico ha riguardato infatti anche gli uomini [Žarkov 2001; United Nations 1994, 56].

Questo contribuì a creare la convinzione serba dello "stupro nazionalista”, in altri termini la politicizzazione dello stupro, visto come parte di un complotto albanese per costringere i serbi ad andarsene dal Kosovo [Bracewell 2000, 563-565]. Il nazionalismo serbo venne presto imitato dal regime croato di Franjo Tudjman, ed entrambi si rifacevano ai movimenti nazionalisti ottocenteschi che collegavano la mascolinità $\mathrm{e}$ le politiche espansionistiche aggressive dell'epoca imperialista attraverso 
la metafora della nazione madre e della santità e inviolabilità del corpo della patria, le cui frontiere dovevano essere protette dalla violenza altrui [Blom, Hagemann, Hall 2000; Banti 2005a; Banti 2005b].

$\mathrm{Si}$ trattava, in altre parole, di un nazionalismo di genere costruito dalla commistione di genere e sessualità con razza, etnicità e classe [YuvalDavis 1997]. Ne conseguì che l'uso nazionalista della violenza di genere, di solito definita come etnica, divenne uno strumento per passare sotto silenzio l'esplosione di pretese nazionaliste - non solo nella ex Jugoslavia, ma anche in altre svariate terre alla fine della Guerra fredda - attraverso un processo di «homogenizacia nacije», l'omogeneizzazione di ogni singola nazione. Questo processo mirava a nascondere, dunque, le svariate ragioni per le tensioni economiche e sociali riconducendole alla sola causa etnica e a slogan retorici [Janigro 1999, 20].

Simile fu quanto accadde nei primi anni Novanta in Ruanda, dove il nazionalismo e la preparazione del genocidio si affinarono attraverso la sessualizzazione dei corpi delle donne. I media, in particolare, crearono una propaganda basata sull'odio per le donne del gruppo nemico: il giornale Kangura pubblicò nel dicembre del 1990 un articolo intitolato Richiamo alla coscienza degli Hutu, la cui quinta parte conteneva i tristemente noti cosiddetti Dieci comandamenti Hutu che istruivano gli Hutu a marginalizzare, disumanizzare e quindi sterminare il nemico - identificato con $\mathrm{i}$ Tutsi in patria, con $\mathrm{i}$ Tutsi fuoriusciti a seguito della presa del potere nel 1973 del dittatore Hutu Juvénal Habyarimana e organizzati nel Fronte Patriottico Rwandese e infine con gli Hutu moderati che tentavano di riportare la democrazia nel paese. La costruzione politica del nemico era infatti iniziata con la decolonizzazione, ed era basata su teorie razziali razziste importate dalle potenze occidentali durante l'epoca coloniale tedesca e belga, e dai missionari. Gli europei avevano infatti costruito la propria relazione con gli abitanti locali su supposte differenze biologiche tra Hutu e Tutsi, creando in questo modo e quindi cristallizzando, attraverso l'istituzione di un'identità etnica basata su carte di identità per i locali, la divisione tra i due gruppi [Vidal 1996, 336-337]. Prima della 
colonizzazione, infatti, la definizione di Tutsi e di Hutu era semplicemente socioeconomica [Mukagasana 2011, 18].

Il primo dei Dieci comandamenti attaccava le donne Tutsi viste al servizio del "nemico" (ovvero i Tutsi che combattevano la dittatura rwandese dal loro esilio all'estero, i Tutsi nel paese accusati di sostenerli, e gli Hutu moderati ritenuti troppo critici nei confronti del governo razzista rwandese), e altri quattro "comandamenti" riguardavano le donne e la sessualità. La quarta parte dell'articolo raffigurava le donne Tutsi come strumento sessuale usato dagli uomini Tutsi per distruggere gli uomini Hutu, ammaliandoli e irretendoli [ICTR-99-52-T, 45-47]. Le donne Tutsi erano descritte con la parola Ibizungerezi, che in Kinyarwanda significa «bellissime e sexy» [Human Rights Watch 1996, 16]. Vi erano poi nella stampa svariati fumetti, destinati a un pubblico illetterato, $i$ quali dipingevano le donne Tutsi come oggetto sessuale a disposizione degli uomini Hutu, i quali venivano incitati a stuprarle per far perdere loro la supposta arroganza, e per "assaggiarle". Altri mostravano le donne Tutsi come sessualmente impegnate con il generale ONU Roméo Dallaire, o raffiguravano il Primo ministro Agathe Uwilingiyimana nuda e a letto con altri politici, o con serpi che le uscivano dal seno [Chrétien 2002, 336, 368; ICTR-99-52-T, 68-69].

Tutto ciò preparò la via alla futura violenza, che non risparmiò neppure donne non Tutsi, non solo le Hutu moderate, ma più genericamente ragazze molto giovani e molto belle, senza tenere in considerazione alcuna la loro appartenenza etnica [de Brouwer 2005, 13]. La sessualizzazione dei corpi delle vittime ebbe come conseguenza anche l'uso diffuso della tortura e di un sadismo volto a mutilare parti del corpo, come i seni quali simbolo femminile, o il naso sottile e le dita affusolate considerati tipici dei Tutsi secondo un diffuso stereotipo di epoca coloniale [Bruneteau 2004, 227, 237]. 


\section{Lo stupro di massa per attaccare il futuro di un popolo}

In Bosnia come in Ruanda, lo stupro di massa fomentato dal nazionalismo non fu usato solo come arma diretta di guerra, ma anche con lo scopo di attaccare il futuro del gruppo nemico tentando di impedirne la normalizzazione postbellica, attraverso i "figli del nemico" in Bosnia [Di Palma 2011] e la diffusione intenzionale di HIV e di AIDS in Ruanda. Da qui la seconda ragione della comparazione tra i due paesi: in entrambi, i conflitti degli anni Novanta usarono lo stupro di massa e la violenza contro le donne secondo una prospettiva qualitativamente diversa. Questo fu in parte riconosciuto dalla giurisprudenza internazionale: i due tribunali penali internazionali ad hoc, quello per la ex Jugoslavia (International Criminal Tribunal for the Former Yugoslavia, ICTY) e per il Ruanda (ICTR) hanno compiuto passi importanti, stabilendo che lo stupro e il genocidio erano correlati: il massacro di Srebrenica fu genocidio [ICTY IT-98-33-A, 87]; lo stupro venne usato in Bosnia per costringere la popolazione ad andarsene [U.N S/1994/674/ Add.2 1994, 10] e come crimine contro l'umanità [ICTY IT-96-23T\&23/1-T, 281-283]; lo stupro fu atto di genocidio in Ruanda a Taba, dove «la violenza sessuale fu parte integrale del processo di distruzione [...] del gruppo Tutsi nel suo complesso. [...] distruzione dello spirito, della volontà di vivere, e della vita stessa» [ICTR-96-4-T, 176-177].

I tribunali non stabilirono tuttavia la correlazione innovativa, secondo quanto qui sostenuto, tra violenza fisica e simbolica sul corpo delle donne e lo scopo di lasciare una ferita permanente nel dopoguerra di questi conflitti. In Ruanda, per prevenire il recupero futuro della popolazione nemica, le donne furono stuprate, deliberatamente infettate con l'HIV e mantenute in vita [Sperling 2006, 645; Sharlach 2002, 117; Donovan 2002 , s17]. Lo stupro di massa organizzato nei campi di stupro o nelle cosiddette "stanze delle donne" in Bosnia fu a sua volta qualcosa di nuovo; le sue origini si trovavano sia nel vecchio concetto della donna come simbolo della nazione, sia nella costruzione nuova dello stato nazione attraverso lo stupro come arma bellica incisa sui corpi delle donne. 
Costringere le donne stuprate a generare bambini ha un valore simbolico potente: le donne diventano "strumento di comunicazione» tra gruppi di uomini aggressori e i loro nemici sulla definizione delle frontiere [Iveković 1999, 75]. Lo stupro mirava a produrre «bambini di un'altra religione» [Héritier 1996, 15] o bambini della «etnia del nemico» [U.N S/1994/674/Add.2 1994, 11] e fu usuale/normale/sistematico [Nahoum-Grappe 1996, 192]. Le Nazioni Unite stabilirono inoltre che donne di tutti i gruppi nazionali furono stuprate nel corso delle guerre di dissoluzione della ex Jugoslavia, ma non c'è alcuna «equivalenza morale nell'analisi» [U.N S/1994/674/Add.2 1994, 9]: lo stupro delle donne musulmane di Bosnia fu usato come strategia militare attraverso la diffamazione simbolica della cultura e della religione del nemico - con l'analogia, ad esempio, della deliberata crudeltà e umiliazione nell'uccidere le vittime tagliando loro la gola con un gancio da macellaio [Goytisolo 2001, 22], come fossero bestie da macellare.

Ci fu dunque un'auto riproduzione della violenza, con una violenza patriarcale, strutturale, di base, su cui poté innestarsi una nuova violenza fisica e simbolica. Sia in Bosnia sia in Ruanda, l'esplosione della violenza contro le donne portò infatti con sé violenza fisica sessuale e violenza simbolica, mostrando come nella strategia della guerra contro le donne il controllo diretto sul corpo non sia necessario: è sufficiente un controllo indiretto attraverso il corpo, una dominazione di potere ottenuta plasmando la relazione tra le donne e la loro comunità attraverso gravidanze forzate, come in Bosnia, o l'infezione di HIV e AIDS, come in Ruanda.

Di frequente le donne stuprate furono infatti detenute in Bosnia fino a quando non poterono più abortire [Cacic-Kumpes 1995, 13; Rezun 1995, 161] e veniva detto loro che dovevano mettere al mondo un figlio "cetnico» [Thomas e Ralph 1999, 204], come emerge ad esempio dalle parole di una donna detenuta nel campo di concentramento serbo di Omarska: «Mi dissero [...] che dovevo generare un serbo - allora sarei stata diversa anch'io» [Helsinki Watch 1993, 164]. Questo nasce dalla 
concezione patriarcale dell'appartenenza di un bambino al gruppo etnico del padre [Fisher 1996-97, 114], mentre l'identificazione dei serbi con il gruppo nazionalista cetnico deriva dalla Seconda guerra mondiale [United Nations 1994, 59], quando il nome creato per designare i soldati serbi in lotta contro gli ottomani iniziò ad identificare i soldati jugoslavi, perlopiù serbi, fedeli al monarca jugoslavo (esiliato dall'occupante nazionalsocialista) e impegnati nella lotta contro la Wehrmacht, ma anche contro i partigiani comunisti di Josip Broz Tito e contro i nazionalisti croati ùstascia, nella formazione dell'Esercito jugoslavo in patria.

Come sottolinea Salzman, «il mito genetico culturale e patriarcale» è condiviso da tutti i gruppi nazionali in causa, e «la stessa pratica di stuprare e di mettere incinta le donne come forma di genocidio deriva non solo da come i perpetratori fanno proprio il mito genetico e culturale, ma anche da come le vittime, le loro famiglie e le loro comunità accettano, a loro volta, tale mito» [Salzman 1998, 365]. I carnefici sapevano che nella cultura musulmana una donna non vergine e stuprata non è maritabile e non può essere una madre, e che anche nella sua stessa famiglia una donna violata è spesso rifiutata dal marito. K.S. di Ključ afferma in proposito: "La donna lo tiene segreto. È una vergogna. Pertanto. La madre lo nasconde quando è accaduto alla figlia in modo che possa sposarsi, e viene nascosto anche se è accaduto ad una donna più matura, per preservare il matrimonio» [Helsinki Watch 1993, 178]. Proprio come le gravidanze forzate furono la strategia bellica contro la futura ripresa in Bosnia, così le infezioni deliberate delle donne stuprate con HIV e AIDS furono attuate in Ruanda, dove a molte donne i perpetratori dissero che «sarebbero morte lentamente e di una morte estenuante per AIDS» [Sharlach 2000, 99]. La presenza massiccia di stupratori infetti da HIV, che contagiarono le donne intenzionalmente e non, causò una rapida crescita del numero di vittime positive all'HIV: almeno il 67 per cento delle donne Tutsi stuprate contrasse infatti il virus [Amnesty International 2004, 3]. Questo fu un altro modo di usare la violenza sia sessuale sia simbolica sulle donne, pensate come contenitori, in questo caso non del figlio del nemico ma della malattia altrui, allo scopo non 
solo di contagiare le donne ma anche di impedire la ripresa della loro società di appartenenza nel dopoguerra, come conseguenza del conflitto e del genocidio. Neppure gli uomini furono risparmiati dalle infezioni volute, come nel caso di P. il quale fu costretto ad un rapporto sessuale con una donna ritenuta positiva all'HIV [African Rights 2004, 19].

Come conseguenza dello stupro di massa, anche in Ruanda crebbe il numero di bambini nati dallo stupro, ma qui non si trattò del risultato di una politica di gravidanze forzate [Sharlach 2000, 100]. Tuttavia, gli effetti a lungo termine sulla società, nonché sulla reazione materna nei confronti dei bambini - "bimbi di stupro" o "figli dell'odio" come vennero chiamati in Bosnia, enfants de mauvais souvenir (bambini dei cattivi ricordi) o "bambini non voluti" in Ruanda - furono simili in entrambi i paesi: quando le donne non poterono abortire, generarono figli che, come riportano diversi operatori sanitari, spesso furono uccisi alla nascita, di frequente abbandonati in orfanotrofi o dati in adozione [Human Rights Watch 1996, 4], e raramente accettati dalle madri e da loro cresciuti.

Come sottolinea la scrittrice femminista Slavenka Drakulić, «le conseguenze dell'accettazione di un bambino nato da stupro sono serie. Il bambino avrà, in un certo senso, un'identità completamente falsa e di questo la madre ne sarà responsabile» [Drakulić 1999, 8]. Ma nell'esistenza spesso solitaria delle sopravvissute a stupro, i bambini poterono anche divenire la loro unica compagnia, come nel caso di C.: "Quando restai incinta, inizialmente mi vergognavo. Ma oggi devo ammettere che questa bambina è la mia unica gioia. L'ho chiamata Umumararungu Diane, colei che cura la mia solitudine» [Mukagasana e Kazinierakis 2008, 62]. 


\section{II dopoguerra: il silenzio e nuove violenze}

I figli nati da stupro furono solo una delle conseguenze che colpirono le donne nel dopoguerra dei conflitti in Bosnia e in Ruanda. Ci furono infatti almeno quattro grossi problemi che le sopravvissute a stupro dovettero fronteggiare, tutti connessi al silenzio imposto e tutti portatori di nuove violenze su queste donne: un supporto psicologico e sanitario inadeguato per persone che, prima di tutto, avevano bisogno di parlare e di essere ascoltate e quindi curate; la mancanza di giustizia, una protezione legale carente, sia dei diritti civili delle donne sia contro violenze future; infine una scarsa attenzione per le narrative e le memorie delle sopravvissute all'interno della nuova identità collettiva nazionalista postbellica.

Questi problemi ebbero come risultato l'inefficacia dei programmi di recupero, che divennero invece spesso un altro modo di mettere a tacere i sopravvissuti, negando loro comprensione della violenza che avevano sofferto e ignorando il loro bisogno di un diverso approccio nell'analizzare la loro situazione, al di là della richiesta di dimenticare per contribuire al processo di pace, o dell'usarli di nuovo come simbolo della nazione violata [Mannergren Selimovic 2010, 57-61].

Si segue qui l'idea di Siri Hustvedt secondo la quale «tutti i pazienti hanno storie, e queste storie sono indubbiamente parte del significato della loro malattia» [Hustvedt 2010, 36], e si ritiene quindi che lasciare che le donne raccontino le proprie storie sia già parte del processo di sostegno psicologico cui spesso le donne hanno un accesso limitato. L'assistenza sanitaria, sia fisica sia psicologica, è infatti fornita ai sopravvissuti solo parzialmente e minata dalla stigmatizzazione familiare e comunitaria, dallo status economico e dalla provenienza geografica dei pazienti, e soprattutto si basa su una diversa e discriminante valutazione della violenza sessuale rispetto ad altri traumi di guerra.

Le sopravvissute a stupro in Bosnia hanno un difficile accesso alle coperture assicurative, e le donne che non possono affrontare i costi delle cure mediche o che vivono in aree remote sono di fatto escluse [CEC 
2008, 18; Avdibegovic et. al. 2008] e marginalizzate per quanto riguarda l'assistenza legale. Nella Republika Srpska, una sopravvissuta a violenza sessuale deve certificare un danno fisico pari o superiore al 60 per cento per accedere all'assicurazione medica e alle cure, mentre per i veterani di guerra è sufficiente una certificazione del 20 per cento di danno fisico [RS Law N. 25/93, n. 46/04 e 53/04 Art. 4]. Nella Federazione di Bosnia e Herzegovina, le vittime della violenza sessuale in guerra sono equiparate alle vittime civili [FBiH Law n. 39/06 Art. 5] e i civili sono discriminati rispetto ai veterani di guerra, ricevendo un rimborso massimo del 70 per cento rispetto a quanto accordato ai veterani [FBiH Law n. 39/06 Art. 9].

Poche donne ricevono un trattamento adeguato, e poche donne si fidano delle istituzioni e chiedono aiuto sia in Bosnia e Herzegovina [Klarić et al. 2007] sia in Ruanda [Mukamana e Brysiewicz 2008; Human Rights Watch 1996], dove la situazione è aggravata dalla diffusione di HIV e AIDS durante e dopo il genocidio, non solo perché molte donne furono stuprate ed infettate, ma anche a causa delle condizioni igieniche carenti e della mancanza di infrastrutture adeguate nel dopoguerra [African Rights 2004, 30; UNAIDS/WHO 2008, 11-12].

Lo stupro causò in Ruanda non solo danno fisico immediato, ma anche conseguenze psicologiche e fisiche a lungo termine, e più della violenza sessuale in sé, l'infezione all'HIV è legata alla stigmatizzazione e alla marginalizzazione sociale ed economica. Molte donne non possono affrontare i costi delle cure per l'AIDS [Mukagasana e Kazinierakis 2008, 10], e nel timore del risultato di un test dell'HIV e del giudizio della comunità di appartenenza, diverse sopravvissute non vanno neppure a fare il test, come ricorda A.: "Non ho mai fatto il test; peggiorerebbe soltanto la mia situazione» [African Rights 2004, 50]. I. conferma i pregiudizi contro le donne stuprate ed infettate dall'HIV: «Non sono accettata nella mia comunità $[. .$.$] la gente è molto cattiva con me» [de Brouwer \mathrm{e}$ Hon Chu 2009, 83], mentre G. fu persino costretta ad andarsene di casa, rifiutata dalla famiglia [de Brouwer e Hon Chu 2009, 110]. Molti uo- 
mini sono sospettosi e ritengono che le sopravvissute abbiano in qualche modo collaborato con i perpetratori allo scopo di aver salva la vita [Amnesty International 2004, 7; Gourevitch 2000, 232], e rifiutano le donne stuprate o non vogliono intraprendere una relazione con una ragazza stuprata durante nel genocidio - anche a causa della stigmatizzazione dello stupro in una società in cui per tradizione la donna è sottomessa al marito e alla sua famiglia [Polidori 2009, 169], e della vedova accusata della morte del consorte [Gbikpi 2006]. Ad esempio M.O. racconta che il suo compagno ha accettato di iniziare ad uscire con lei dopo che lei gli ha giurato, mentendo, di non essere stata violentata durante il genocidio [de Brouwer e Hon Chu 2009, 39].

Questo è solo uno dei problemi, ma in entrambi i case studies ve ne sono molti altri di tipo psicologico e fisico. «Se non fosse per il sostegno psicologico e le medicine, sarei morta. [...] Non posso dormire senza pillole. [...] Ho bisogno di aiuto", confessa T., nella Republika Srpska, ad anni di distanza dalla fine della guerra [Amnesty International 2009, 3]. I traumi delle donne riguardano principalmente sindrome da stress post-traumatico, disagi psichici (depressione, ansia, somatizzazione, difficoltà nei rapporti sessuali, insonnia) e problemi somatici (a livello cardiovascolare, ma anche diabete, problemi alla tiroide, sindrome psico organica, problemi scheletro muscolari e difficoltà al tratto urogenitale) [African Rights 2004]. Come afferma il direttore dell'ospedale centrale di Kigali, Dr. Emile Rwamasirabo, le vittime di stupro non cercano cure mediche perché si vergognano troppo, mentre il dottor Etienne Mubarutso, ginecologo presso l'ospedale universitario di Butare, sottolinea: «si cura la malattia diretta, ma psicologicamente non vengono guarite. Continuano a tornare lamentando crampi e dolori, ma non hanno alcun problema fisico. Queste donne sono profondamente segnate a livello psicologico» [Human Rights Watch 1996, 72-73].

Le donne nascondono la propria sofferenza e nella maggior parte dei casi tacciono, sentendo che le cure ricevute sono inadeguate ad attenuare il loro dolore e che la società in cui vivono, e persino la loro stessa 
famiglia, difficilmente capirebbero - come nel caso di Zilhada, che non poté ricongiungersi ai figli sopravvissuti nel timore del loro giudizio [Doni e Valentini 1993, 34], o di una tredicenne curata nell'ospedale da campo a Gornji Rahić nel comune di Brčko nel 1993: «interrogata da un ginecologo, anche lui ex prigioniero del campo di concentramento di Brčko, la ragazzina tentò di nascondere lo stupro, e inventò di essere sposata» [Bukvić 2007, 23].

Un secondo, grave problema fronteggiato dalle donne che hanno subito violenza sessuale concerne la giustizia. Le donne che hanno testimoniato, sia davanti ai due tribunali penali internazionali per la ex Jugoslavia e per il Ruanda sia presso le corti locali, sono spesso minacciate e intimidite; non vengono adeguatamente protette e la loro identità è stata spesso resa pubblica. Le donne di Bosnia si sentono abbandonate, dato che i loro ex carnefici possono facilmente conoscere la loro vera identità quando testimoniano ai processi, e non beneficiano di alcun rimborso economico per aver corso dei rischi andando a testimoniare [Lombezzi 2006], mentre il programma di protezione dei testimoni dell'ICTY non segue le donne al loro ritorno a casa. Alcuni criminali sono ancora liberi e impuniti, e questo rafforza la convinzione delle donne che non ci sia giustizia. In Ruanda, diversi sopravvissuti sono stati picchiati o uccisi da ex carnefici [de Brouwer e Hon Chu 2009, 105, 123], e Amnesty International riporta, dopo una visita al tribunale di Arusha alla fine del 1997, che «il programma di protezione dei testimoni è debole e di scarsa efficacia. Mette i testimoni a rischio, scoraggia le persone a rendere testimonianza e mette a repentaglio la giustizia» [Amnesty International 1998, 6].

«Vivere con i perpetratori di un genocidio è una grande sfida per tutti noi sopravvissuti», afferma M.C., riguardo al fatto che i testimoni ad Arusha e alle corti locali dei gacaca sono ben noti a quanti hanno accusato con la loro testimonianza [de Brouwer e Hon Chu 2009, 71] e i sopravvissuti non si sentono al sicuro. Oltre a ciò, molti carnefici vengono liberati e minacciano le donne che li hanno accusati, e per questo alcuni, 
come J., affermano: «Non vado più ai gacaca, perché le persone che ho accusato vengono rilasciate e non capisco il senso di correre il rischio di rilasciare la mia testimonianza se non fa alcuna differenza» [de Brouwer e Hon Chu 2009, 56]. P. aggiunge che «i gacaca portano più lacrime che sorrisi» [de Brouwer e Hon Chu 2009, 77]. Anche l'indennizzo economico non è sufficiente, se non giunge insieme al pentimento: alcune donne sono umiliate dagli ex carnefici che cercano di "comprare" il perdono della propria vittima in cambio di denaro o di beni, senza sentirsi peraltro davvero in colpa e pentiti [de Brouwer e Hon Chu 2009, 63].

I processi per crimini sessuali sono pochi rispetto alla mole dei testimoni. Per esempio, nel processo contro Milan Lukić, ex comandante di un'armata paramilitare serba, lo stupro non fu nemmeno incluso tra i capi d'accusa nonostante diverse donne avessero testimoniato di essere state stuprate da lui in persona a Višegrad [ICTY IT-98-32/1-T, 2007, 18]. L'ICTR è stato criticato invece per aver sottovalutato i testimoni, come nel processo a Sylvestre Gacumbitsi, il borgomastro (la più alta carica a livello locale) del comune di Rusumo - l'imputato fu giudicato colpevole di genocidio e di crimini contro l'umanità, ma fu assolto da tre capi d'accusa di stupro dalla Camera d'Appello, la quale ritenne vi fosse insufficienza di prove a causa della scarsa credibilità dei testimoni [ICTR-2001-64-A, 34-35; Rushing et al. 2006, 38-39]. Una capacità di indagine scarsa, e una conseguente impossibilità di incriminare per violenza sessuale, portarono ad uno scandalo nel cosiddetto processo Cyangugu - in cui, nonostante le prove, non fu incluso alcun capo d'accusa per stupro. Quando al processo alcune vittime testimoniarono di essere state stuprate, il giudice stabilì che la corte non potesse prendere in considerazione le prove per un crimine che non compariva tra le imputazioni [Nowrojee 2005, 14-17]; il processo si chiuse nel 2004 con l'assoluzione di due imputati su tre [ICTR-99-46-T, 208].

Il terzo problema fronteggiato dalle sopravvissute è la mancanza di una protezione statale legale adeguata, non solo sulla violenza di genere, ma anche nell'implementazione dei diritti delle donne. Sebbene questo sia 
una questione aperta per tutte le donne in generale, le sopravvissute sono più vulnerabili, come evidenziano alcuni dati del 2005 per il Ruanda: il 31 per cento delle donne che hanno subito violenza sessuale è stata soggetta a violenza domestica dopo il genocidio, perlopiù per mano del partner, perché era troppo malata, debole o psicologicamente sottoposta a minacce per lasciare la propria casa o rifiutare rapporti sessuali [NISR $2006,177]$. Anche in Bosnia, la violenza di genere non è tenuta in grande considerazione nella complessa situazione postbellica, caratterizzata da gravi violazioni dei diritti umani, soprattutto per quanto riguarda il diritto alla proprietà e al ritorno nelle proprie case - come stabilito dalla Law property implementation del 2000 dell'Office of the High Representative (OHR), organismo istituzionale ad hoc responsabile dell'implementazione degli accordi di Dayton nella loro parte civile. Tale situazione ricade sulla discriminazione femminile nella disposizione delle proprietà, nella reintegrazione socioeconomica dopo la restituzione delle abitazioni, nell'accesso al mondo del lavoro e nella violenza domestica «prevalente, non sufficientemente indagata e non abbastanza denunciata» [Refugee Women's Resource Project and Asylum Aid 2002, 15].

C'è infatti un legame diretto tra l'incremento della violenza domestica e la guerra da poco conclusa, a causa di problemi nella reintegrazione delle famiglie, dell'accresciuta violenza tra gli uomini combattenti durante il conflitto e dello stress post-traumatico, di fronte alla maggiore debolezza delle donne stuprate. Tuttavia, "non si è verificato un incremento parallelo nelle strutture sociali o giuridiche volte a proteggere dalle violazioni in questi diritti umani elementari» [Open Society Institute 2007, 11], nonostante per legge i diritti delle donne siano garantiti nella Federazione di Bosnia e Herzegovina - con una legge sull'uguaglianza tra generi nella sfera pubblica e privata e contro la discriminazione basata sul genere [O.G. 16/03] emanata dopo anni di lotte da parte di Ngo femministe, e con una legge sulla protezione dalla violenza domestica [O.G. 22/05, 51/06], mentre l'articolo 222 del Codice Penale sanziona la «violenza nella famiglia» [O.G. 6/03, 37/03, 21/04, 69/04, 18/05]. 
La Republika Srpska, a sua volta, punisce la violenza domestica, come riportato nel Codice penale [O.G. 22/00] e nella legge sulla violenza domestica [art. 208, O.G. n. 118/05, 17/08], ma le donne soggette a violenza domestica non sono riconosciute come aventi diritto a misure di protezione sociale, e le Case di Accoglienza per donne che aiutano le vittime non sono riconosciute come enti di protezione sociale [Udružene 2008].

In maniera analoga, in Ruanda c'è un dislivello notevole tra il sistema giuridico e l'implementazione delle leggi. La Costituzione del 2003 (art. 10, 11, 16, 46 e 47) protegge tutti i cittadini dalla discriminazione [We-Actx for Hope 2007, 2-3]. Nel paese vi è stato un lungo dibattito parlamentare sulla violenza di genere [Draft Law 2006], sfociato in una legge sulla prevenzione della medesima e sull'incriminazione dei perpetratori [Legge 59/2008]. Il governo ha istituito un telefono amico e un Ufficio di Monitoraggio per il Genere [We-Actx for Hope 2007, 54-63]; diversi sono gli “sportelli per la violenza di genere" nelle stazioni di polizia e nell'esercito, e di recente il Ministro per la Promozione di Genere e Famiglia ha creato un Piano Nazionale Strategico per il Genere [MIGEPROF 2009].

La Legge rwandese sul Genocidio del 2004 (una delle tante Leggi sul Genocidio passate dal Parlamento dal 1996) riconosce lo stupro e la violenza sessuale come atti di genocidio, mentre la Legge sul Genocidio del 2008 assicura alle testimoni dei gacaca consulenti esperti in trauma [de Brouwer e Hon Chu 2009, 151], ma l'inefficacia di questo tipo di tribunale locale, nonché l'assenza di compensazione economica, pone a rischio le condizioni di vita delle sopravvissute. La stigmatizzazione sociale nella comunità di appartenenza, la mancanza di giustizia, l'inadeguatezza delle cure sanitarie, problemi economici e una nuova vittimizzazione da parte degli ex perpetratori hanno indotto diverse donne a prostituirsi in cambio di cibo [Amnesty International 2004, 9].

La messa a tacere è infine legata al quarto problema delle vittime di stupro: lo sfruttamento delle loro storie. Per quanto riguarda la ex Ju- 
goslavia, questa strategia non fu funzionale solo al mito prebellico dello stupro attuato dal nemico e alla propaganda dei media durante i conflitti [Cockburn 2001], quando ogni donna violata fu rapidamente trasformata nel simbolo della nazione violentata, nonché nell'uso nazionalista delle donne sul tema del ritorno nel focolare domestico [Drakulić 1993] o riguardo la chiamata nazionalista ad essere madri per la patria [Bracewell 1996] secondo la credenza patriarcale per la quale la maternità è il ruolo naturale della donna [Yuval-Davis 1996]. Ora, infatti, la nuova memoria pubblica e collettiva postbellica, incentrata sugli uomini e patriarcale, usa le donne stuprate per costruire un nuovo dopoguerra nazionalista [Kesić 2003, 011, 043], con la "guerra dei numeri" attuato da ogni gruppo nazionale a proposito delle proprie vittime di stupro, ritenute più numerose di quelle degli altri gruppi, e una crescente commiserazione vittimista, senza sottolineare invece il ruolo attivo di reazione delle donne alla violenza e la loro prospettiva anti-nazionalista non solo sulla violenza subita ma anche sulle strategie per affrontare la vita nel dopoguerra.

Come scrive Andrea Petö a proposito degli stupri perpetrati dai soldati dell'Armata rossa alla fine della Seconda guerra mondiale in Ungheria, «nei modelli a volte conflittuali della narrazione dello stupro, troviamo diversi sistemi di verità a seconda dell'epoca e del narratore» [Petö 2003, 131]. La narrazione postbellica sulla Bosnia sfrutta l'argomento dello stupro, ancora una volta, per incrementare il numero delle proprie vittime [Žarkov 1999] senza ascoltarle davvero né rendere loro giustizia.

\section{Il dopoguerra: strategie di reazione}

Mantenere le donne nel silenzio, o vittimizzarle per utilizzarne la violenza subita ai fini della propaganda postbellica, sono due aspetti dello stesso atteggiamento paternalista attuato nei loro confronti, contro il quale una prima forma di consapevolezza consiste nella rottura del si- 
lenzio per riconquistare l'autostima innanzitutto, e secondariamente per creare una memoria condivisa. Il ricordo (e di riflesso il racconto) infatti può essere "sovversivo» [Richter 2003, 42] per chi vuole manipolare la memoria [Janigro 2003]. Lo esprime bene tra gli altri E. scegliendo di raccontare ad un'altra sopravvissuta: "Abbiamo una storia simile e per questo mi fido di te» [Mukagasana, Kazinierakis 2008, 22].

Non a caso, una prima fase di narrazione ha riguardato la creazione di gruppi di ascolto per donne - l'ascolto come pratica del femminismo [Richter 2003, 53] - che hanno subito violenza, e solo in un secondo tempo l'apertura della testimonianza ad un pubblico comunque selezionato e in un certo senso fidato - secondo il principio della pedagogia degli oppressi: «la liberazione: non un dono, non una conquista propria, ma un processo in mutuo svolgimento» [Freire 1970, 43-72].

Se alcune donne riescono ad affrontare il silenzio, per quelle che trovano ancora troppo arduo parlare intervengono narrazioni giornalistiche e novelle basate su storie vere, come quella della giornalista Slavenka Drakulić scaturita da riflessioni di fronte ad una donna che aveva subito violenza: «Desiderava raccontare ma le risultava impossibile farlo...Non riusciva a smettere di tremare. Capii allora per la prima volta che la sua storia era proprio in quello che non riusciva a dire. E dovevo trovare un modo per raccontare io al posto suo» [Drakulić 1999, 3-4, corsivo mio]. Mosse da riflessioni analoghe sull'incapacità di diverse donne a mettersi a nudo in prima persona attraverso il racconto, diverse organizzazioni e associazioni femministe hanno iniziato a raccogliere testimonianze di vittime di violenza nella guerra di dissoluzione della Jugoslavia, spesso opponendosi ai nuovi nazionalismi attraverso gruppi di donne di diverse nazionalità, come già avevano prima del conflitto [Žarkov 1999], quali ad esempio Donne in Nero di Belgrado il Network delle Donne della Ex Jugoslavia. La loro azione è volta da un lato a reinserire narrative marginalizzate nella memoria collettiva, dall'altro a demolire lo stereotipo della donna come vittima passiva di stupro e, nel caso delle donne di Bosnia, come vittime musulmane facili della società rurale patriarcale. 
Con il loro lavoro, tali gruppi sottolineano invece come le donne abbiano vissuto esperienze che vanno oltre il loro essere corpi in guerra [Kašić 2000], come alla violenza abbiano saputo reagire [Helms 2003, 96], e come a ben vedere le vittime della violenza maschile siano donne e basta, indipendentemente da qualsiasi connotazione nazionale o religiosa [Richter 2003, 23-24].

Sia in Ruanda sia in Bosnia, infine, la risposta alla negazione dei diritti di genere in guerra ha comportato la nascita di gruppi di donne dediti al miglioramento delle condizioni di vita a livello locale e a combattere la discriminazione postbellica, da Pro-femmes Twese Hamwe, una piattaforma rwandese che raggruppa diverse associazioni, a Medica Zenica in Bosnia - già attiva nell'accoglienza delle donne stuprate e rilasciate dai campi di concentramento a gravidanza avanzata, e che si dichiara, non a caso, femminista e anti-nazionalista [Helms 2003,117] e che opera nella delicata sovrapposizione tra passata violenza di guerra e violenza postbellica domestica o perpetrata dagli ex carnefici del conflitto [Refugee Women's Resource Project and Asylum Aid 2002, 29].

Diversi sono infatti gli episodi di donne maltrattate e abusate psicologicamente e fisicamente da mariti che le accusano dello stupro subito in guerra [Amnesty International, 2009, 59], e fornire loro assistenza e aiuto significa per i gruppi di donne e per le associazioni femministe farne, da vittime di stupro in guerra e dopo, cittadine consapevoli dei propri diritti e contrarie alla "domesticazione" nei ruoli tradizionali di protettrici della casa e della famiglia [Rener 2003, 69].

\section{Conclusioni}

I diversi fattori che concorrono, in entrambi i case studies, a vittimizzare nuovamente le donne che hanno subito violenza sessuale durante i conflitti degli anni Novanta in Bosnia e in Ruanda invitano a far riflettere sui meccanismi di intervento che entrambe le società devono attuare 
per implementare i diritti delle donne e sollevano alcune questioni sulla violenza di genere in sé, indipendentemente dal contesto bellico.

Sebbene ragioni di spazio non permettano di soffermarsi sulla violenza sessuale in quanto tale, e quindi sulla condivisione delle tradizioni patriarcali nel rapporto tra $i$ generi dominante anche in altre aree del mondo e in contesti di pace, vale la pena di ricordare, in breve, come la violenza sessuale sia possibile all'interno della violenza strutturale propria di tutte le società a diffuso potere patriarcale, in cui il corpo femminile è concepito in relazione all'uso che gli uomini ne fanno [Corbin 1989, VII]. Il possesso del corpo permette di stabilire precise gerarchie, e non è mera violenza fisica ma anche simbolica, mezzo per affermare il dominio maschile, attraverso comportamenti stereotipati nella costruzione sociale della virilità attraverso il corpo dell'uomo. La violenza sessuale dipende dunque da come la mascolinità si rapporta al potere [Giddens 2006, 819] e riveste un significato che va oltre la concezione del corpo della donna come proprietà maschile [Brownmiller 1993, 24] e che si connota come socio-culturale nella definizione di barriere e di ruoli [Shorter 1977].

Sulla base di tali considerazioni, si comprende come l'aggressione sessuale vada collocata in rapporto al potere attraverso l'esibizione della virilità dell'aggressore, in contesti in cui oggetto della violenza sono quanti esulano dal modello virile e omofobo dominante - principalmente le donne, ma anche uomini appartenenti a categorie discriminate quali omosessuali, travestiti, detenuti [Vigarello 1998, 248-9].

Negli ultimi decenni è cresciuta l'attenzione internazionale per la violenza sessuale come arma di guerra, a fronte di un interesse meno marcato per le forme di violenza di genere più 'quotidiane'. Sembra che l'attenzione alla violenza di guerra porti a minimizzare sia forme più diffuse di violenza, sia la somiglianza tra la violenze contro le donne non solo in guerra ma anche dopo, come i casi del Ruanda e della Bosnia mostrano. La crescente attenzione alla violenza di genere in guerra riflette probabilmente un'accresciuta consapevolezza delle conseguenze sociali 
di tale violenza per la società nel suo complesso, piuttosto che un timore per l'impatto della violenza sulle donne in sé e per sé. Diversi studiosi hanno applicato il concetto di "continuum della violenza" alla questione della distinzione tra guerra e pace [Cockburn 2004], chiedendosi se la violenza contro le donne sia davvero diversa dalla "normale" violenza di genere, o piuttosto un mero esacerbarsi della stessa, e se la violenza di guerra sia peggiore della "normale" violenza nel dopoguerra, per concludere che "non c'è dopoguerra per le donne. La violenza continua ad esserci perché le sue cause sottese rimangono intatte» [Pillay 2001]. Tale continuum è stato chiamato in causa, non a caso, anche durante le guerre balcaniche degli anni Novanta, sulla questione se lo stupro fosse un problema di sicurezza collettiva o individuale [Hansen 2001]. Vedere la violenza sessuale come arma di guerra è comunque utile a svelare l'uso politico e strategico della violenza stessa come tattica di dominio [Boesten 2010]: se le comunità delle vittime acquisissero tale consapevolezza, le donne potrebbero evitare di tenere in privato la colpa e il danno [Sideris 2001]. Tuttavia, fino a quando la consapevolezza della violenza sessuale come tattica di dominio è saldata al contesto bellico piuttosto che allo stupro in sé, il mancato riconoscimento della natura politica della violenza ne fa il mezzo più potente per dominare le donne in tempo di pace. Solo una già citata nuova "filosofia del femminismo» [Kesić 2003, 049], partendo dalle esperienze delle donne con una presa di posizione forte contro la violenza di genere in sé, non solo riguardo la giustizia e la protezione delle vittime ma anche nella implementazione della legislazione interna, può avviare il sovvertimento della concezione patriarcale delle donne come cittadine inferiori. 


\section{Fonti}

\section{Legislazione}

International Criminal Court, Rome Statute, art. 7 (Crimes against Humanity), comma g, 4.

United Nations Security Council Resolution 1820 (2008) on women and peace and security (S/RES/1820) (2008).

Codice Penale della Federazione di Bosnia e Herzegovina, Official Gazette of Federation BiH, n. 36/03, 37/03, 21/04, 69/04, 18/05.

FBiH (Federazione di Bosnia e Herzegovina), Law on Gender Equality, Official Gazette of Federation BiH, n. 16/03.

$\mathrm{FBiH}$, Law on Amendments and Additions to the Law on the Basis of the Social Protection, Protection of Civilian Victims of War and Families with Children in the FBiH, Official Gazette of Federation BiH, n. 39/06, Article 5, Article 9.

FBiH, Law on Protection from Domestic Violence of the Federation of Bosnia and Herzegovina, Official Gazette of Federation BiH, n. 22/05 and 51/06.

Codice penale della Republika Srpska, Official Gazette of the Republika Srpska, n. 22/00.

Codice penale della Republika Srpska, art. 208, Official Gazette of the Republika Srpska, n. $118 / 05,17 / 08$.

RS (Republika Srpska), Law on Protection of Civilian Victims of War, Official Gazette of the Republika Srpska, N. 25/93, 30 December 2003 with later amendments, Article 2.

RS, Law on the Rights of Servicemen, Military Invalids and Families of the Deceased Servicemen of the Defence of Motherland War in Republika Srpska, Official Gazette of the Republika Srpska, n. 46/04 and 53/04, Article 4.

MIGEPROF (Ministry of Gender and Family Promotion) 2009, Terms of Reference for National and International Consultancy, Consultancy for the elaboration of the Policy on GBV and the development of a Strategic \& operational Plan for implementation, Kigali: Migeprof (570 06/19/2009).

Rwandan Parliament 2006, Draft Law on the Prevention, Protection and Punishment of Any Gender Based Violence, Draft Law of 3 August 2006.

Rwandan Law 59/2008 of 10/09/2009 on Prevention and Punishment of Gender based Violence.

\section{Report e statistiche}

African Rights 2004, Rwanda. Broken Bodies, Torn Spirits. Living with Genocide, Rape and HIV/AIDS, Kigali: African Rights. 
Amnesty International 1998, International Criminal Tribunal for Rwanda. Trials and Tribulations, London: Amnesty International (IOR 40/003/1998).

- 2004, Rwanda: "Marked for Death", Rape Survivors Living with HIV/AIDS in Rwan$d a$, London: Amnesty International (AFR 47/007/2004).

- 2009, 'Whose Justice?' The Women of Bosnia and Herzegovina are Still Waiting, London: Amnesty International (EUR 63/06/2009).

CEC (Commission of the European Community) 2008, Bosnia and Herzegovina 2008 Progress Report. Commission Staff Working Document Accompanying the Communication from the Commission to the European Parliament and the Council. Enlargement Strategy and Main Challenges 2008-2009, $\operatorname{COM(2008)~} 674$.

Helsinki Warch 1993, War Crimes in Bosnia-Hercegovina, vol. 2, New York: Human Rights Watch.

Human Rights Watch 1996, Shattered Lives. Sexual Violence during the Rwandan Genocide and its Aftermath, New York: Human Rights Watch.

NISR (National Institute of Statistics of Rwanda) 2006, Rwanda Demographic and Health Survey 2005, Kigali: NISR.

Open Society Institute 2007, Violence Against Women. Does the Government Care in Bosnia and Herzegovina?, Budapest: Open Society Institute.

Refugee Women's Resource Project and Asylum Aid 2002, Refugee Women and Domestic Violence: Country Studies. Bosnia and Herzegovina, London: Refugee Women's Resource Project and Asylum Aid.

Udružene Banja Luka, Helsinki Parlament građana Banja Luka 2008, Open letter to the People's Assembly of Republika Sprska, Government of Republika Srpska, Ministry of Health and Social Protection of Republika Srpska, Board of Equal Possibilities of the People's Assembly of Republika Srpska, Gender Center of RS Government, Banja Luka, Bosnia and Herzegovina.

UNAIDS/WHO 2008, Epidemiological Fact Sheets on HIV and AIDS. Core Data on Epidemiology and Response. Rwanda, 2008 update, Geneva.

United Nations 1994, Final Report of the Commission of Experts Established Pursuant to Security Council Resolution 780(1992), New York: United Nations Organization (S/1994/674).

WE-ACTx for Hope 2007, Know your Rights!, Kigali: WE-ACTx for Hope.

\section{Atti dei Tribunali penali internazionali ad hoc, ICTY e ICTR}

ICTR-96-4-T: Akayesu (Judgement), 2 September 1998 (Prosecutor v. Jean-Paul Akayesu).

ICTR-99-52-T: Nahimana et al. (Judgement and Sentence), 3 December 2003 (Prosecutor v. Ferdinand Nahimana, Jean-Bosco Barayagwiza, Hassan Ngeze). 
ICTR-99-46-T: Ntagerura et a. (Judgement and Sentence), 25 February 2004 (Prosecutor v. André Ntagerura, Emanuel Bagambiki and Samuel Imahishimwe).

ICTR-2001-64-A: Gacumbitsi (Appeal Judgement), 7 July 2006 (Sylvestre Gacumbitsi v. Prosecutor).

ICTY IT-96-23-T\&23/1-T: Kunarać et al. (Judgement), 22 February 2001 (Prosecutor v Dragoljub Kunarać, Radomir Kovač and Zoran Vuković).

ICTY IT-98-32/1-T: Lukić at al (Judgement), 20 July 2009 (Prosecutor v Milan Lukić and Sredoje Lukić).

ICTY IT-98-33-A: Krstić (Appeal), 19 April 2004 (Prosecutor v Radislav Krstić).

\section{Bibliografia}

Agosín M. (ed.) 2001, Women, Gender, and Human Rights: A Global Perspective, New Brunswick, NJ: Rutgers University Press.

Avdibegovic E. et al. 2008, Mental Health Care of Psychotraumatized Persons in PostWar Bosnia and Herzegovina. Exepriences from Tuzla Canton, "Psychiatria Danubina», XX (4): 474-484.

Banti A.M. 2005a, Corpi e confini nell'immaginario nazional-patriottico ottocentesco, in Salvatici S. (ed.) 2005, Confini. Costruzioni, attraversamenti, rappresentazioni, Soveria Mannelli: Rubbettino, 199-218.

- 2005b, L'onore della nazione. Identità sessuali e violenza nel nazionalismo europeo dal XVIII secolo alla Grande Guerra, Torino: Einaudi.

Bartoloni S. (ed.) 2002, A volto scoperto. Donne e diritti umani, Roma: Manifestolibri.

Beevor A. 2007, Berlin: The Downfall 1945, London: Penguin Books.

Bianchi B. 2008, Crimini di guerra e crimini contro l'umanità durante la Grande Guerra. Le stragi sul fronte orientale, in Procacci G., Silver M., Bertuccelli L. 2008, Le stragi rimosse. Storia, memoria pubblica, scritture, Milano: Unicopli, 19-39.

Blom I., Hagemann K., Hall C. (eds.) 2000, Gendered Nations. Nationalisms and Gender Order in the Long Nineteenth Century, Oxford-New York: Berg Publishers.

Boesten J. 2010, Analyzing Rape Regimes at the Interface of War and Peace in Peru, "The International Journal of Transitional Justice», 4: 110-129.

Bourke J. 2007, Rape. Sex, Violence, History, Berkeley, CA: Counterpoint; trad. Cavallo M.G., Fantoni L., Falcone P. 2009: Stupro. Storia della violenza sessuale, Roma-Bari: Laterza.

Bracewell W. 1996, Women, Motherhood, and Contemporary Serbian Nationalism, «Women's Studies International Forum», 19 (1-2): 25-33.

- 2000, Rape in Kosovo: masculinity and Serbian nationalism, «Nations and Nationalism», 6 (1): 563-590. 
Brownmiller S. 1993, Against Our Will. Men, Women and Rape, New York: Ballantine Books (I ed. 1975).

Brown W. 1995, States of Injury: Power and Freedom in Late Modernity, Princeton, NJ: Princeton University Press.

Bruneteau B. 2004, Le Siècle des genocides; trad. Flores D’Arcais A. 2005, Il secolo dei genocidi, Bologna: il Mulino.

Bukvić E. 2007, Una guerra contro le donne e le future generazioni. Stupro etnico nella Bosnia Erzegovina, «Difesa sociale», 2: 19-40.

Cacic-Kumpes J. 1995, War, Ethnicity, and Violence Against Women, "Refuge», 14 (8): 12-15.

Chrétien J.P. (ed.) 2002, Rwanda, les médias du génocide, Paris: Karthala.

Cockburn C. 2001, The Gendered Dynamics of Armed Conflict and Political Violence, in Moser C.O.N., Clark F.C. (eds.) 2001, Victims, Perpetrators or Actors? Gender, Armed Conflict and Political Violence, London and New York: Zed Books, 13-29.

- 2004, The Continuum of Violence: A gender perspective on War and Peace, in Giles W.M., Hyndman J. (eds.) 2004, Sites of Violence: Gender and Conflict Zones, London: University of California Press, 24-44.

Copelon R. 1995, Gendered War Crimes: Reconceptualizing Rape in Time of War, in Perter J., Wolper A. (eds.), Women's Rights, Human Rights. International Feminist Perspectives, New York: Routledge, 197-214.

Corbin A. (ed.) 1989, Violences sexuelles; trad. Garin M. 1993: La violenza sessuale nella storia, Roma-Bari: Laterza.

Dallaire R. 2003, J'ai serré la main du diable. La faillite de l'humanité au Rwanda, Montréal: Libre Expression.

de Brouwer A.M. 2005, Supranational Criminal Prosecution of Sexual Violence. The ICC and the Practice of the ICTY and the ICTR, Antwerpen-Oxford: Intersentia.

de Brouwer A.M., Hon Chu S.K. (eds.) 2009, The Men Who Killed Me. Rwandan Survivors of Sexual Violence, Vancouver: Douglas \& McIntyre.

Derderian K. 2005, Common Fate, Different Experience: Gender-Specific Aspects of the Armenian Genocide, 1915-1917, "Holocaust and Genocide Studies», 19 (1): 1-25.

Diken B., Laustsen, C.B. 2005, Becoming Abject: Rape as a Weapon of War, "Body \& Society», 11 (1): 111-128.

Di Palma S. 2011, Guerra e violenza di genere. Gli stupri in Bosnia negli anni Novanta del Novecento, "Contemporanea», 3: 457-477.

Doni E., Valentini C. 1993, L’arma dello stupro. Voci di donne della Bosnia, Palermo: La Luna.

Donovan P. 2002, Rape and HIV/AIDS in Rwanda, "The Lancet Supplement», 360 (9350): 17-18. 
Drakulić S. 1993, Women and the New Democracy in the Former Yugoslavia, in Funk N., Muller M. (eds.) 1993, Gender Politics and Post-Communism. Reflections from Eastern Europe and the Former Soviet Union, London: Routledge, 123-130.

- 1999, An Introduction to S. A Novel About the Balkans, in Eadem, Kao da me nema; trad. Ivić M. 1999, As If I am Not There, ritradotto 2001, S. A Novel about the Balkans, New York: Penguin Books, 1-8; trad. Leto M.R. 2000, Come se io non ci fossi, Milano: Rizzoli.

Fisher S.K. 1996-97, Occupation of the Womb: Forced Impregnation as Genocide, "Duke Law Journal», 91: 91-133.

Flores M. (ed.) 2010, Stupri di guerra. La violenza di massa contro le donne nel Novecento, Milano: FrancoAngeli.

Freire P. 1970, Pedagogia do oprimido; trad. Bergman R.M. 2006, Pedagogy of the Oppressed, New York: Continuum.

Gaeta P. 2006, La repressione penale dei crimini internazionali. Problemi e prospettive, in Calloni M. 2006, Violenza senza legge. Genocidi e crimini di guerra nell'età globale, Torino: Utet, 143-158.

Gbikpi B. 2006, Ruanda: passi verso la riconciliazione, in Calloni M. 2006, Violenza senza legge. Genocidi e crimini di guerra nell'età globale, Torino: Utet, 102-120.

Giddens A. 2006, Gender and Crime, in Idem, Sociology, Cambridge, Polity Press: 815-819.

Glover G. 2001, Humanity. A Moral History of the Twentieth Century, New Haven, CT: Yale University Press.

Gourevitch P. 2000, We wish to inform you that tomorrow we will be killed with our families. Stories from Rwanda, London: Picador; trad. Gobetti N, 2000, Desideriamo informarla che domani verremo uccisi con le nostre famiglie. Storie dal Ruanda, Torino: Einaudi.

Goytisolo J. 2001, Landscapes of War. From Sarajevo to Chechnya, San Francisco, CA: City Lights Publishers.

Green J.L. 2004, Uncovering Collective Rape. A Comparative Study of Political Sexual Violence, «International Journal of Sociology», 34 (1): 97-116.

Grill B. 2005, Il genocidio negato. Ruanda, l'invenzione della crudeltà, in Idem, Africa!, Roma: Fandangolibri, 227-250.

Hansen L. 2001, Gender, Nation, Rape: Bosnia and the construction of security, «International Feminist Journal of Politics», 3 (1): 55-75.

Helms E. 2003, Women as Agents of Ethnic Reconciliation? Women's Ngos and International Intervention in Postwar Bosnia-Herzegovina, "Women's Studies International Forum», 26 (1): 15-33.

Héritier F. 1996, Réflexions pour nourrir la réflexion, in Idem (ed.) 1996, De la violence, Paris: Editions Odile Jacob; trad. Pacelli L. 1997, Sulla violenza, Roma: Meltemi, 


\section{1-53.}

Hustvedt S. 2010, The Shaking Woman or a History of My Nerves, London: Sceptre.

Iveković R. 1999, Une autopsie des Balkans. Essai de psycho-politique; trad. Prezzo R. 1999, Autopsia dei Balcani. Saggio di psico-politica, Milano: Raffaello Cortina Editore.

Janigro N. 1999, L'esplosione delle nazioni. Le guerre balcaniche di fine secolo, Milano: Feltrinelli.

- 2003, In bilico fra modernità e barbarie nel paese dei destini incrociati, in Richter M., Bacchi M. (ed.) 2003, Le guerre cominciano a primavera. Soggetti e genere nel conflitto jugoslavo, Soveria Mannelli: Rubettino, 77-94.

Kaldor M. 1999, New and Old Wars. Organized Violence in a Global Era, Stanford, CA: Stanford University Press; trad. Foglia G. 1999: Le nuove guerre. La violenza organizzata nell'età globale, Roma: Carocci.

Kašić, B. 2000, The aesthetic of the victim within the discourse of war, in Slapšak S. (ed.) 2000, War Discourse, Women's Discourse: Essays and Case-Studies from Yugoslavia and Russia, Ljubljana: Topos, 271-283.

Kesić V. 2003, Women Reconstructing Memories, in Kesić V., Janković V., Bijelić B. 2003, Women Recollecting Memories. The Center for Women War Victims Ten Years Later, Second Expanded Edition of the 1994 CWWV Collected Papers, Zagreb: Centar za Žene Žrtve Rata, 007-012.

- 2003, Women are Victims of War. But Women Also Know 1.000 Ways to Survive. Women Can Change the World!, in Kesić V., Janković V., Bijelić B. 2003, Women Recollecting Memories. The Center for Women War Victims Ten Years Later, Second Expanded Edition of the 1994 CWWV Collected Papers, Zagreb: Centar za Žene Žrtve Rata, 042-050.

Klarić M. et al. 2007, Psychological Consequences of War Trauma and Postwar Social Stressors in Women in Bosnia and Herzegovina, "Croatian Medical Journal», 48 (2): 167- 176.

Lombezzi M. 2006, Foča, nel lager delle bosniache, «Diario», XI (9): 58-59.

Macedo D. 2000, Introduction to the Anniversary Edition, in Freire P. 2000, Pedagogia do oprimido; trad. Bergman R.M. 2006, Pedagogy of the Oppressed, New York: Continuum, 11-28 (I ed. 1970).

Mannergren Selimovic J. 2010, Remembering and Forgetting After War. Narratives of Truth, Justice and Reconciliation in a Bosnian Town, Gothenburg: Univeversity of Gothenburg.

Marcus S. 1992, Fighting Bodies, Fighting Words: A Theory and Politics of Rape Prevention, in J. Butler J., Scott J. (eds.) 1992, Feminists Theorize the Political, New York: Routledge, 385-403.

Mardorossian C.M. 2002, Toward a New Feminist Theory of Rape, «Signs: Journal of Women in Culture and Society», 27 (3): 743-775. 
Marzo Magno A. (ed.) 2001, La guerra dei dieci anni. Jugoslavia 1991-2001, Milano: Net.

Melander E., Öberg, M., Hall, J. 2009, Are 'New Wars' More Atrocious? Battle Severity, Civilians Killed and Forced Migration Before and After the End of the Cold War, "European Journal of International Relations», XV (3): 505-536.

Menon R., Bhasin K. 1998, Borders and Boundaries: Women in India's Partition, New Brunswick, NJ: Rutgers University Press.

Menon R. (ed.) 2004, No Woman's Land. Women From Pakistan, India And Bangladesh Write on The Partition of India, New York: Women Unlimited.

Mukagasana Y., Kazinierakis A. 2001, Les blessures du silence. Témoignages du génocide au Rwanda; trad. Di Genio L. 2008, Le ferite del silenzio. Testimonianze sul genocidio del Rwanda, Molfetta: Edizioni La Meridiana.

Mukagasana Y. 2011, Un giorno vivrò anch'io. Il genocidio del Rwanda raccontato ai giovani, Molfetta: Edizioni La Meridiana.

Mukamana D., Brysiewicz P. 2008, The Lived Experience of Genocide Rape Survivors in Rwanda, "Journal of Nursing Scholarship», 40 (4): 379-384.

Nahoum-Grappe V. 1996, L'usage politique de la cruauté: l'épuration ethnique (ex-Yougoslavie, 1991-1995), in Héritier F. (ed.) 1996, De la violence; trad. Pacelli L. 1997, Sulla violenza, Roma: Meltemi, 190-227.

Nowrojee B. 2005, "Your Justice is Too Slow". Will the ICTR Fail Rwanda's Rape Victims?, "United Nations Research Institute for Social Development (UNRISD) Occasional Paper», 10.

Petö A. 2003, Memory and the Narrative of Rape in Budapest and Vienna in 1945, in Bessel R., Schumann D. (eds.) 2003, Life After Death. Approaches to a Cultural and Social History of Europe During he 1940s and 1950s, Cambridge: Cambridge University Press, 129-148.

Pillay A. 2001, Violence against Women in the Aftermath, in Meintjies S., Pillay A., Turshen M. (eds.) 2001, The Aftermath: Women in Post-Conflict Transformation, London: Zed Books, 35-45.

Polidori F. 2009, Il detto e il non detto. Violenza e memoria tra le vittime tutsi (19591994), in Fusaschi M. (ed.) 2009, Rwanda: etnografie del post-genocidio, Roma: Meltemi, 156-174.

Ramet S.P. 2006, The Three Yugoslavias. State-Building and Legitimation, 1918-2005, Bloomington, IN: Indiana University Press.

Rener T. 2003, Nazionalismo e donne nelle società post-socialiste, in Richter M., Bacchi M. (eds.) 2003, Le guerre cominciano a primavera. Soggetti e genere nel conflitto jugoslavo, Soveria Mannelli: Rubettino, 61-76.

Rezun M. 1995, Europe and the War in the Balkans: Toward a New Yugoslavian Identity, Westport, CT: Praeger Publishers. 
Richter M. 2003, Introduzione, in Richter M., Bacchi M. (eds.) 2003, Le guerre cominciano a primavera. Soggetti e genere nel conflitto jugoslavo, Soveria Mannelli: Rubettino, 19-57.

Rieff D. 1996, Slaughterhouse. Bosnia and the Failure of the West, New York: Touchstone.

Romani C. 1994, State Responsibility Goes Private: A Feminist Critique of the Public/ Private Distinction in International Human Rights Law, in Cook R.J. (ed.) 1994, Human Rights of Women: National and International Perspectives, Philadelphia: University of Pennsylvania Press, 85-115.

Rossi-Doria A. 2004, Diritti umani e diritti della donne, "Contemporanea", 4: 531-554.

Rumiz P. 2011, Maschere per un massacro. Quello che non abbiamo voluto sapere della guerra in Jugoslavia, Milano: Feltrinelli (I ed. 1996).

Rushing E.J. et al. 2006, Updates from the International Criminal Courts, «Human Rights Brief", 14 (1): 38-43.

Salvatici S. 2007, Donne e diritti umani, in Flores M. (ed.) 2007, Diritti umani. Cultura dei diritti e dignità della persona nell'epoca della globalizzazione, Atlante vol. I, I soggetti e i temi, Torino: Utet, 314-357.

Salzman T.A. 1998, Rape Camps as a Means of Ethnic Cleansing: Religious, Cultural, and Ethical Responses to Rape Victims in the Former Yugoslavia, "Human Rights Quarterly», 20 (2): 348-378.

Sanasarian E. 1989, Gender Distinction in the Genocidal Process: A Preliminary Study of the Armenian Case, "Holocaust and Genocide Studies», 4 (4): 449-461.

Scaglione D. 2010, Istruzioni per un genocidio. Rwanda: cronache di un massacro evitabile, Formigine: Infinito Edizioni.

Sekulić T. 2002, Violenza etnica. I Balcani tra etnonazionalismo e democrazia, Roma: Carocci.

- 2006, Crimini di guerra, crimini contro l'umanità e genocidio: strategie dello "State building» etno-nazionale, in Calloni M. 2006, Violenza senza legge. Genocidi e crimini di guerra nell'età globale, Torino: Utet, 121-135.

Sharlach L. 2000, Rape as Genocide: Bangladesh, the Former Yugoslavia, and Rwanda, «New Political Science», 22 (1): 89-102.

- 2002, State Rape. Sexual Violence as Genocide, in Worcester K., Bermanzohn S.A., Ungar M. (eds.) 2002, Violence and Politics. Globalization's Paradox, London: Routledge, 107-123.

Shorter E. 1977, On Writing the History of Rape, «Signs. Journal of Women in Culture and Society» 3 (2): 471-482.

Sibomana A. 1998, J'accuse per il Rwanda. Ultima intervista a un testimone scomodo, Torino: Ega Editore. 
Sideris T. 2001, Rape in War and Peace: Social Context, Gender, Power and Identity, in Meintjies S., Pillay A., Turshen M. (eds.), The Aftermath: Women in Post-Conflict Transformation, London: Zed Books, 46-62.

Sperling C. 2006, Mother of Atrocities: Pauline Nyiramasuhuko's Role in the Rwandan Genocide, «Forhman Urban Law Journal», XXXIII (2): 637-664.

Strauss S. 2006, The Order of Genocide. Race, Power, and War in Rwanda, Ithaca and London: Cornell University Press.

Thomas D.Q., Ralph R.E. 1999, Rape in War: The Case of Bosnia, in Ramet S.P. 1999, Gender Politics in the Western Balkans. Women and Society in Yugoslavia and the Yugoslav Successor States, University Park, PA: Pennsylvania State University Press, 203218.

Tilly C. 2003, The Politics of Collective Violence, Cambridge: Cambridge University Press.

Vidal C. 1996, Génocide des Tutsi Rwandais: Cruauté délibérée et logique de haine, in Héritier F. (ed.) 1996, De la violence, Paris: Editions Odile Jacob; trad. Pacelli L. 1997, Sulla violenza, Roma: Meltemi, 325-366.

Vigarello G. 1998, Histoire du viol. XVI-XX siècle; trad. Folin A. 2001, Storia della violenza sessuale. XVI-XX secolo, Venezia: Marsilio.

Vitucci C. 2007, I crimini contro le donne nel diritto internazionale, in Fiume G. (ed.) 2007, Donne diritti democrazia, Roma: XL Edizioni, 83-120.

Woodward S.L. 1995, Balkan Tragedy. Chaos and Dissolution after the Cold War, Washington DC: The Brookings Institution.

Yuval-Davis N. 1996, Women and the Biological Reproduction of "the Nation", "Women's Studies International Forum», 19 (1-2): 17-24.

- 1997, Gender and Nation, London: Sage.

Žarkov D. 1999, From 'Media War' to Ethnic War. The Female Body and the Production of Ethnicity in Former Yugoslavia (1986-1994), Nijmegen, NL: University of Nijmegen.

- 2001, The Body of the Other Man. Sexual violence and the Construction of Masculinity, Sexuality and Ethnicity in Croatian Media, in Moser C.O.N., Clark F.C. (eds.) 2001, Victims, Perpetrators or Actors? Gender, Armed Conflict and Political Violence, London and New York: Zed Books, 69-82.

- 2007, The Body of War. Media, Ethnicity, and Gender in the Break-up of Yugoslavia, Durham and London: Duke University Press. 